\title{
p38 MAPK SIGNAL PATHWAY INVOLVED IN ANTI-INFLAMMATORY EFFECT OF CHAIHU-SHUGAN-SAN AND SHEN-LING-BAI-ZHU-SAN ON HEPATOCYTE IN NON-ALCOHOLIC STEATOHEPATITIS RATS
}

\section{QinHe Yang $^{\star 1}$, YongJian Xu ${ }^{1}$, GaoFei Feng ${ }^{1}$, ChaoFeng Hu ${ }^{2}$, YuPei Zhang ${ }^{1}$, ShaoBing Cheng ${ }^{1}$, YanPing Wang ${ }^{2}$, XiangWen Gong ${ }^{1}$}

\author{
${ }^{1}$ Department of Traditional Chinese Medicine, Medical College of Jinan University, 601 Huangpu Road West, \\ Guangzhou, Guangdong, China, 510632. ${ }^{2}$ Department of Pathophysiology, Medical College of Jinan University, 601 \\ Huangpu Road West, Guangzhou, Guangdong, China, 510632 \\ *Email: tyangqh@jnu.edu.cn
}

\begin{abstract}
Background: Traditional Chinese Medicine (TCM), has over thousands-of-years history of use. Chaihu-Shugan-San (CSS), and Shen-ling-bai-zhu-San (SLBZS), are famous traditional Chinese herbal medicine formulas, which have been used in China, for the treatment of many chronic diseases.

Materials and Methods:This study investigated the anti-inflammatory effects of CSS and SLBZS on signaling molecules involved in p38 mitogen-activated protein kinase (p38 MAPK), pathway on hepatocytes of non-alcoholic steatohepatitis (NASH), rats induced by high fat diet. SD male rats were randomly divided into 8 groups: negative control group, model control group, high (9.6g/kg/day)/low (3.2g/kg/day)-dose CSS group, high (30g/kg/day)/low (10g/kg/day)-dose SLBZS group, high (39.6g/kg/day)/low (13.2g/kg/day)-dose integrated group. The rats of NASH model were induced by feeding a high-fat diet. After 16, wks, Hepatocytes were isolated from 6 , rats in each group by collagenase perfusion. The liver histopathological changes and serum inflammatory cytokines TNF- $\alpha$, IL-6 were determined. The proteins of TLR4, phosphor-p38 MAPK and p38 MAPK involved in p38 MAPK signal pathway were assayed.

Results: The statistical data indicated the NASH model rats reproduced typical histopathological features of NASH in human. CSS and SLBZS ameliorated lipid metabolic disturbance, attenuated NASH progression, decreased the levels of TNF- $\alpha$ and IL-6 in serum, as well as inhibited TLR4 protein expression, p38 MAPK phosphorylation, and activation of p38 MAPK. In conclusion, CSS and SLBZS might work as a significant anti-inflammatory effect on hepatocyte of NASH by inhibiting the activation of TLR4, p-p38 MAPK and p38 MAPK involved in p38 MAPK signal pathway.
\end{abstract}

Conclusion: To some extent, CSS and SLBZS may be a potential alternative and complementary medicine to protect against liver injury, alleviate the inflammation reaction, moderate NASH progression.

Key words: p38 mitogen-activated protein kinase; Toll like receptor 4; Hepatocytes; Non-alcoholic Steatohepatitis; Traditional Chinese medicine;

Non-standard Abbreviations: Chaihu-Shugan-San (CSS); Shen-ling-bai-zhu-San (SLBZS)

\section{Introduction}

Non-alcoholic steatohepatitis (NASH), is a chronic process liver disease characterized by hepatocellular ballooning degeneration and necroinflammation based on hepatic steatosis (Angulo, 2002; Vanni et al., 2010). It is within an important stage from simple steatosis development to fibrosis, and cirrhosis in non-alcoholic fatty liver disease (NAFLD) (Kopec et al., 2011). Past studies indicated that the excessive inflammatory cytokines such as TNF- $\alpha$ (Yimin et al., 2012; Tilg et al., 2000; Kudo et al., 2009; Choi et al., 2005), and IL-6 (Tilg et al., 2000; Lalor et al., 2007; Tilg et al., 2010; Ji et al., 2011) exacerbated cell lipid peroxidation and liver injury, promoted NASH progression from different ways.

Toll like receptor (TLR), a kind of membrane receptor protein, is wildly existed in membrane surface of immunological cells and TLR4 is one of the members in the known TLR family. TLR4 is the main receptor in the lipopolysaccharide (LPS)-mediate immune responses (Jin et al., 
http://dx.doi.org/10.4314/ajtcam.v11i1.34

2008). Mitogen-activated protein kinases (MAPKs), are a highly conserved family of serine/threonine kinases incluing known ERK 1/2, JNK/ SAPK, p38MAPK, ERK5/BMK1, which are all important signaling molecules in the control of cellular biological effects to extracellular stimuli. After TLR4 is integrated with LPS, MAPKs cascade reactions are activated and then the p38 MAPK protein is phosphorylated, leading to release of inflammatory factors and starting cell damage mechanism (Takeda et al., 2004; Liu et al., 2010). It was demonstrated that many nosogenesis of inflammatory diseases were mediated with TLR4-p38 MAPK signal pathway (Xu et al., 2010; Tian et al., 2011).

In oriental countries, Traditional Chinese Medicine (TCM), has over thousands-of-years history of use. Chaihu-Shugan-San (CSS), an ancient classical formula from "Jingyue Quanshu" written by Jingyue Zhang of China in 1640 A.D. It is composed of seven Chinese herbs: Bupleurum chinese DC (6g), Pericarpium citri Reticulatae (6g), Ligusticum chuanxiong Hort (5g), Rhizoma Cyperi (5g), Fructus Aurantii (5g), Radix Paeonia Alba (5g) and Glycyrrhiza uralensis Fisch (3g). Shen-ling-bai-zhu-San (SLBZS) is also a famous classical formula recorded in “Taiping Huimin Heji Ju Fang” written by the imperial medical bureau of the Song Dynasty in 1078 A.D. It consists of ten species of medicinal herbs: Panax Ginseng (15g), Atractylodes Ovata (15g), Poria Cocos (15g), Dioscorea Batatasm (15g), Dolichos Lablab (12g), Coix Lachryma-jobi (9g), Nelumbo Nucifera (9g), Glycyrrhiza uralensis Fisch (9g), Platycodon Grandiflorum (6g) and Amomum Xanthioides (6g). Based on the theory of TCM, CSS dredges liver qi, and enhances blood circulation, is prescribed mainly for the liver qi stasis. SLBZS has the functions of tonifying spleen and stomach qi, and is mainly used for deficiency of spleen and stomach.

Many resent studies have demonstrated that CSS protects against lipid peroxidation (Chen et al., 2004; Li et al., 2010), liver fibrosis (Fu et al., 2007; Pang et al., 2009), insulin resistance (Xue et al., 2010). And SLBZS is able to inhibit of activities on oxidative stress (You et al., 2012), lipid peroxidation (Li et al., 2012), and inflammatory reaction (You et al., 2012; Liu et al., 2011). Some of the major compounds from CSS and SLBZS formula, like saikosaponins (Ge et al., 2011; Chiang et al., 2003; Zheng et al., 2011), total glucosides of paeony (Liu et al., 2012; Zhao et al., 2012), ginsenoside (Zeng et al., 2011; Li et al., 2011), atractylenolide (Wang et al., 2012), atractylodes macrocephalaon polysaccharide (Zhang et al., 2011), and Carboxymethylpachymaran (Zhou et al., 2009), also have been identified for their potential protection on liver. Thus, it is essential to investigate the molecular mechanisms of anti-inflammatory effect of CSS and SLBZS to treat NASH. The paper is the first to study the anti-inflammatory effect of CSS and SLBZS on signaling molecules involved in p38 MAPK signal pathway in hepatocytes of NASH.

\section{Materials and Methods \\ Preparation of CSS and SLBZS}

CSS is composed of seven Chinese herbs: Bupleurum Chinese DC (6 g), Pericarpium Citri Reticulatae (6 g), Ligusticum chuanxiong Hort (5 g), Rhizoma Cyperi (5 g), Fructus Aurantii (5 g), Radix Paeonia Alba (5 g) and Glycyrrhiza uralensis Fisch (3 g). SLBZS includes Panax Ginseng (15 g), Atractylodes Ovata (15g), Poria Cocos (15 g), Dioscorea Batatasm (15 g), Dolichos Lablab (12 g), Coix Lachryma-jobi (9 g), Nelumbo Nucifera (9 g), Glycyrrhiza uralensis Fisch (9 g), Platycodon Grandiflorum (6 g) and Amomum Xanthioides (6 g). Integrated recipe contains is the mixture of CSS and SLBZS at a ratio of 1:1. All Chinese medicines were formula granules purchased from Shenzhen Sanjiu Medical Co., Ltd. (1005001S). The formula granules were dissolved in the distilled water, and preserved at $-4^{\circ} \mathrm{C}$.

Animals

112 Sprague-Dawley of Specific Pathogen-Free Male rats (6 weeks old, $200 \mathrm{~g} \pm 20 \mathrm{~g}$ ) were obtained from the Laboratory Animal Research Center of Guangzhou University of TCM (Approval No. SCXK (Yue), 2008-0020), Guangdong province, China. The rats were housed under conditions of controlled temperature $\left(24^{\circ} \mathrm{C} \pm 2{ }^{\circ} \mathrm{C}\right)$ and humidity $(70 \% \pm 10 \%)$ in a 12h-light and 12h-dark cycle (lights on from 8:00 am to 8:00 $\mathrm{pm})$, with free access to diet and water.

\section{Experimental design}

After one week adaptive breeding, the rats were randomly divided into 8 groups, 14 rats in each group: negative control group (NC), model control group (MC), high-dose CSS group (H-CSS), low-dose CSS group (L-CSS), high-dose SLBZS group (H-SLBZS), low-dose SLBZS group (L-SLBZS), high-dose integrated recipe group (H-IG), low-dose integrated recipe group (L-IG). Rat models of NASH were duplicated according to method as our previously reported (Yang et al., 2011) with some minor modifications. Negative control group of rats got free access to a normal chow diet, model control group and treatment groups were fed with high fat diet (HFD), (composed of regular chow $88 \%$, axungia 
http://dx.doi.org/10.4314/ajtcam.v11i1.34

porci $10 \%$, cholesterol $1.5 \%$, bile salt $0.5 \%$ ). All rats in treatment groups were drenched with decoction (H-CSS: $9.6 \mathrm{~g} / \mathrm{kg} / \mathrm{day}, \mathrm{L}-\mathrm{CSS}: 3.2$ g/kg/day, H-SLBZS: $30 \mathrm{~g} / \mathrm{kg} /$ day, L-SLBZS: $10 \mathrm{~g} / \mathrm{kg} / \mathrm{day}, \mathrm{H}-\mathrm{IG}: 39.6 \mathrm{~g} / \mathrm{kg} / \mathrm{day}$, L-IG: $13.2 \mathrm{~g} / \mathrm{kg} / \mathrm{day}$ ), while the rats in the normal group and model group were fed with the same dose $(10 \mathrm{ml} / \mathrm{kg}$ body weight) of distilled water once at 8:00 am everyday. Low-dose equaled human clinical equivalent dosage, and high-dose was 3-fold volume of low-dose. The treatment lasted for 16 weeks.

At the end of treatment, rats in each group were divided into two groups by table of random number: 8, rats for liver samples collection, 6 , rats for isolation of hepatocytes. All rats were treated in compliance with the Guiding Principles for Animal Experiments and the protocols were approved by the Animal Experimental Ethnics Committee of Jinan University, China.

\section{Blood lipid and histopathological examination of liver}

Total cholesterol (TC) and triglyceride (TG) in the serum were determined with automatic biochemical analyzer (Olympus, Japan). The paraffin-embedded liver tissue (about $1 \mathrm{~cm} \times 0.5 \mathrm{~cm} \times 0.5 \mathrm{~cm}$ ) which selected the same part of the liver, about $0.5 \mathrm{~cm}$ from the edge of the right hepatic lobule, were sliced at a thickness of $4 \mu \mathrm{m}$ and examined by hematoxylin-eosin (HE) staining. The steatosis grade, fibrosis stage and inflammation of NASH were evaluated according to the NASH histological scoring system (Kleiner et al., 2005).

\section{Determination of inflammatory cytokines in serum}

The levels of TNF- $\alpha$, IL-6, in serum were measured according to the recommended procedures provided by the enzyme-linked immunosorbent assay (ELISA), kits (TNF- $\alpha$, lot No.E3720-1101-1, Beijing Dake Biotechnology Co., Ltd.; IL-6, lot No.R110407-07A, Shenzhen Xinbo Biotechnology Co., Ltd., China).

\section{Hepatocyte isolation, purification and identification}

Hepatocytes were isolated from 6 rats in each group by collagenase perfusion as described (Hamelet et al., 2009). After 12hrs, incubation at $37^{\circ} \mathrm{C}$, cells were washed three times with PBS. Non-adherent cells were washed off. The hepatocytes viability was $>99 \%$ (as tested by trypan blue dye exclusion). The purity of hepatocytes was $>95 \%$ [as assessed by flow cytometry method using ck-18 antibody according to (Luo et al., 2008)] (Figure 1).

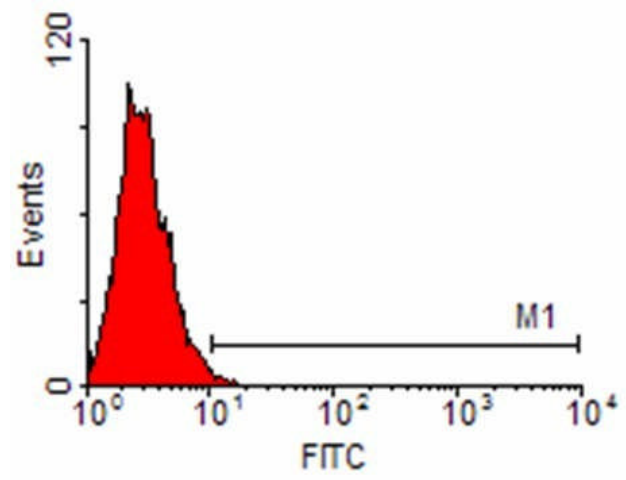

Negative Control

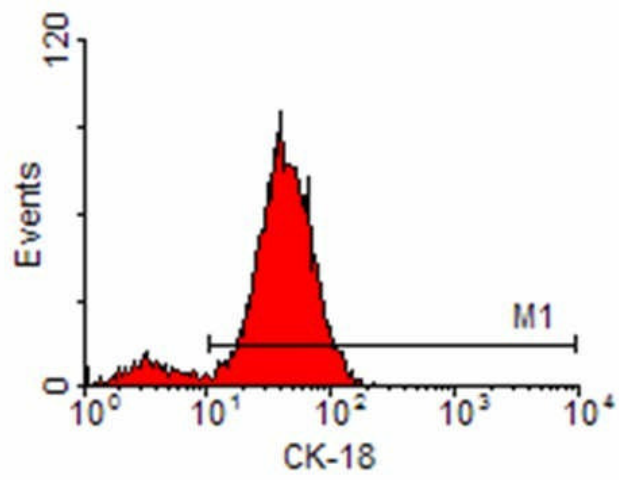

Hepatocyte

Figure 1: The purity of hepatocytes assessed by flow cytometry method

M1: Labeling of low concentration marker, FITC: Fluorescein isothiocyanate. The purity of hepatocytes was >95\% (as assessed by flow cytometry method using anti-ck-18). Negative cell is on the left side of the M1 line. Positive cell (hepatocyte) is inside the M1 line.

\section{Protein extraction and Western blot}

Western blotting was used to determine proteins of hepatocytes TLR4, phosphor-p38 MAPK (p-p38 MAPK), p38 MAPK and Glyceraldehyde-3-phosphate dehydrogenase (GAPDH). GAPDH was used as an internal control. Protein preparations were subjected to 10\% 
http://dx.doi.org/10.4314/ajtcam.v11i1.34

SDS-PAGE and transferred to polyvinylidene difluoride (PVDF) membrane. After transfer, membrane was blocked in $5 \%$ skim milk in Tris-Buffered Saline Tween-20 (1.5 mM Tris, $5 \mathrm{mM} \mathrm{NaCl}, 0.1 \%$ Tween20) and incubated overnight at $4 \square$ with specific primary antibodies. Anti-TLR4 was purchased from Santa Cruz Biotechnology (USA). Anti-p38 MAPK, Anti-phosphor-p38 MAPK and GAPDH antibody were purchased from Cell Signaling Technology (USA). Horseradish peroxidase conjugated goat-anti-rabbit antibody and electro chemical luminescence reagent (Beyotime Biotechnology, China) were used to detect specific proteins. Blots were scanned and densitometry analysis of band intensities was measured with gel image processing system.

\section{Statistical analysis}

The results were expressed as the mean \pm standard deviation (S.D.), unless otherwise indicated. A one-way Analysis of variance (ANOVA) was used to determine the statistical significance of the differences. Probability value $(P)$ less than 0.05 was considered statistically significant. All data were analyzed with the Statistical Package for the Social Sciences (SPSS, USA) 13.0 Software.

\section{Results}

\section{Effects of CSS and SLBZS on serum TC and TG levels and liver histopathological changes}

Elevated serum TC, TG levels and liver histopathological changes indicate hepatic lipid accumulation and lipid metabolic disturbance. As shown in table 1 , the serum TC and TG levels were significantly increased in model group compared to the negative control group $(P<0.01)$. The group of H-CSS, H-SLBZS, L-SLBZS H-IG and L-IG reduced the serum TC and TG levels compared with model control group $(P<0.01$, $P<0.05)$. Liver specimens with HE staining were shown in Figure 2. Sections of liver from model control group scored 12 points had showed typical NASH features, including microvesicular and macrovesicular steatosis, lobular and portal inflammation, and hepatocyte ballooning (Figure 2), and there was a significant difference compared with negative control group $(P<0.01)$. The pathological changes in the treatment groups lightened to different degree as compared with model control group $(P<0.01, P<0.05)$ (Table 2). The results indicated that HFD-induced rats model of NASH had lipid accumulation and lipid metabolic disturbance obviously. CSS and SLBZS ameliorated lipid metabolic disturbance, attenuated NASH progression to some extent.

Table 1: Effects of CSS and SLBZS on serum levels of TC and TG

\begin{tabular}{|l|l|l|}
\hline Group & TG(mmol/L) & TC $(\mathrm{mmol} / \mathrm{L})$ \\
\hline NC & $0.67 \pm 0.23$ & $1.42 \pm 0.29$ \\
\hline MC & $0.79 \pm 0.26^{\mathrm{a}}$ & $3.90 \pm 0.87^{\mathrm{a}}$ \\
\hline H-CSS & $0.60 \pm 0.20^{\mathrm{b}}$ & $3.40 \pm 0.82$ \\
\hline L-CSS & $0.64 \pm 0.13$ & $4.16 \pm 0.63$ \\
\hline H-SLBZS & $0.58 \pm 0.20^{\mathrm{b}}$ & $3.35 \pm 1.46$ \\
\hline L-SLBZS & $0.60 \pm 0.23^{\mathrm{b}}$ & $3.81 \pm 0.81$ \\
\hline H-IG & $0.45 \pm 0.15^{\mathrm{c}}$ & $2.85 \pm 0.51$ \\
\hline L-IG & $0.44 \pm 0.10^{\mathrm{c}}$ & $3.14 \pm 0.39$ \\
\hline
\end{tabular}

Values are means \pm S.D. of 8 rats per group; ${ }^{\mathrm{a}} P<0.01$ compared with $\mathrm{NC}$ group; ${ }^{\mathrm{b}} P<0.05,{ }^{\mathrm{c}} P<0.01$ compared with $\mathrm{MC}$ group

Table 2: NASH histological scoring in different groups

\begin{tabular}{|l|l|}
\hline Group & NASH histological scoring \\
\hline NC & $3.00 \pm 1.58$ \\
\hline MC & $12 \pm 1.87^{\mathrm{a}}$ \\
\hline H-CSS & $9.44 \pm 1.24^{\mathrm{b}}$ \\
\hline L-CSS & $9.51 \pm 1.36^{\mathrm{b}}$ \\
\hline
\end{tabular}


Yang et al., Afr J Tradit Complement Altern Med. (2014) 11(1):213-221

http://dx.doi.org/10.4314/ajtcam.v11i1.34

\begin{tabular}{|l|l|}
\hline H-SLBZS & $5.44 \pm 1.81^{\mathrm{c}}$ \\
\hline L-SLBZS & $6.00 \pm 1.58^{\mathrm{c}}$ \\
\hline H-IG & $5.22 \pm 1.07^{\mathrm{c}}$ \\
\hline L-IG & $7.67 \pm 1.23^{\mathrm{c}}$ \\
\hline
\end{tabular}

Values are means \pm S.D. of 8 rats per group; ${ }^{\mathrm{a}} P<0.01$ compared with $\mathrm{NC}$ group; ${ }^{\mathrm{b}} P<0.05,{ }^{\mathrm{c}} P<0.01$ compared with $\mathrm{MC}$ group
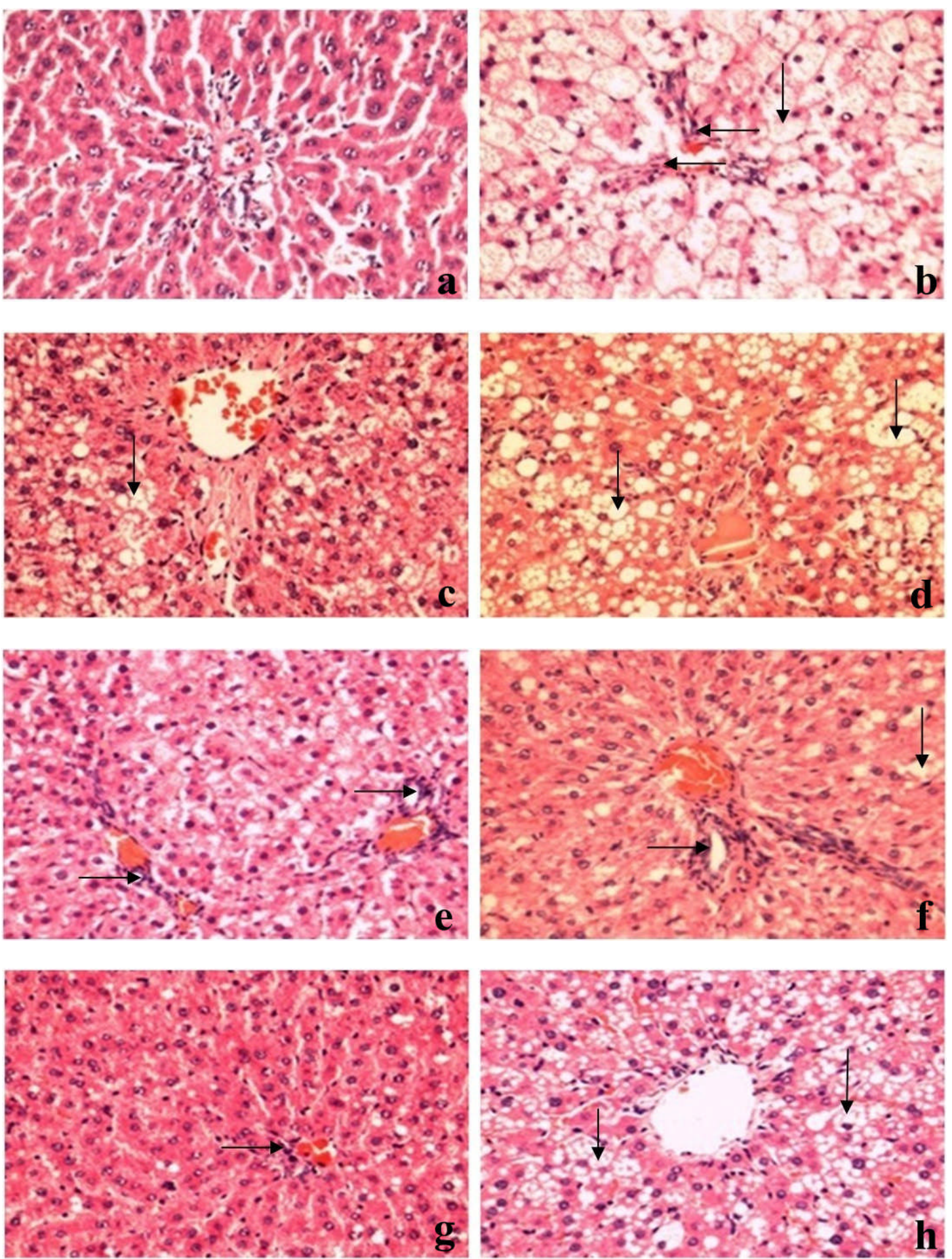

Figure 2: Histological changes of liver sections in different groups (HE staining 200x)

a: Negative control group (NC), b: Model control group (MC), c: High-dose CSS group (H-CSS), d: Low-dose CSS group (L-CSS), e: High-dose SLBZS group (H-SLBZS), f: Low-dose SLBZS group (L- SLBZS), g: High-dose integrated recipe group (H-IG), h: Low-dose integrated recipe group (L-IG). In model control group, liver sections had showed typical NASH features, including microvesicular or macrovesicular steatosis (vertical arrow), lobular and portal inflammation (horizontal arrow). The pathological changes in the treatment groups 
http://dx.doi.org/10.4314/ajtcam.v11i1.34

lightened to different degree as compared with model control group.

\section{Effects of CSS and SLBZS on serum inflammatory cytokine levels}

Rising inflammatory cytokine levels of TNF- $\alpha$ and IL- 6 are regarded as biomarkers of inflammation. Table 3 showed that levels of TNF- $\alpha$ and IL-6 in model control group were higher than that of the negative control group $(P<0.01)$. The treatment groups had lower serum TNF- $\alpha$ and IL-6 levels compared with model control group $(P<0.01, P<0.05)$. It is thus clear that CSS and SLBZS had significant anti-inflammatory effect on the HFD-induced NASH rats in this experiment.

Table 3 Effects of CSS and SLBZS on serum inflammatory cytokine levels

\begin{tabular}{|l|c|c|}
\hline Group & TNF- $\alpha(\mathrm{pg} / \mathrm{ml})$ & IL-6 (pg/ml) \\
\hline NC & $13.57 \pm 4.64$ & $39.28 \pm 28.53$ \\
\hline MC & $28.26 \pm 8.60^{* a}$ & $132.81 \pm 44.22^{* a}$ \\
\hline H-CSS & $18.16 \pm 5.86^{\mathrm{b}}$ & $63.50 \pm 34.51^{\mathrm{b}}$ \\
\hline L-CSS & $20.49 \pm 7.33^{\mathrm{b}}$ & $67.62 \pm 27.03^{\mathrm{b}}$ \\
\hline H-SLBZS & $17.43 \pm 5.29^{\mathrm{b}}$ & $52.81 \pm 21.88^{\mathrm{b}}$ \\
\hline L-SLBZS & $20.09 \pm 6.59^{\mathrm{b}}$ & $60.38 \pm 36.21^{\mathrm{b}}$ \\
\hline H-IG & $15.07 \pm 6.00^{\mathrm{c}}$ & $41.72 \pm 32.24^{\mathrm{c}}$ \\
\hline L-IG & $18.29 \pm 3.57^{\mathrm{b}}$ & $68.35 \pm 25.58^{\mathrm{b}}$ \\
\hline
\end{tabular}

Values are means \pm S.D. of 8 rats per group; ${ }^{\mathrm{a}} P<0.01$ compared with negative control group; ${ }^{\mathrm{b}} P<0.05,{ }^{\mathrm{c}} P<0.01$ compared with model control group

\section{Effects of CSS and SLBZS on p38 MAPK signal pathway related proteins in hepatocytes}

To explore the mechanism of the anti-inflammatory effect of CSS and SLBZS on hepatocytes of NASH rats, we assayed three important proteins of TLR4, p-p38 MAPK and p38 MAPK involved in p38 MAPK signal pathway which is one important mediator in inflammatory response (Alisi et al., 2010; Xu et al., 2010; Tian et al., 2011). Table 4 showed that expression levels of TLR4, p-p38 MAPK and p38 MAPK as well as the ratio of p-p38 MAPK to total p38 MAPK protein in model control group were significantly higher than those in normal control group, $(P<0.01)$. Compared to the model group, all treatment groups inhibited the expression levels of TLR4, p-p38 MAPK and p38 MAPK $(P<0.01$, $P<0.05)$. And the ratio of $\mathrm{p}$-p38 MAPK to total $\mathrm{p} 38$ MAPK protein were decreased obviously $(P<0.01, P<0.05)$. It indicated that $\mathrm{p} 38 \mathrm{MAPK}$ signal pathway may be activated in hepatocytes of NASH rats. The CSS and SLBZS inhibited activation of p38 MAPK signal pathway in different degrees.

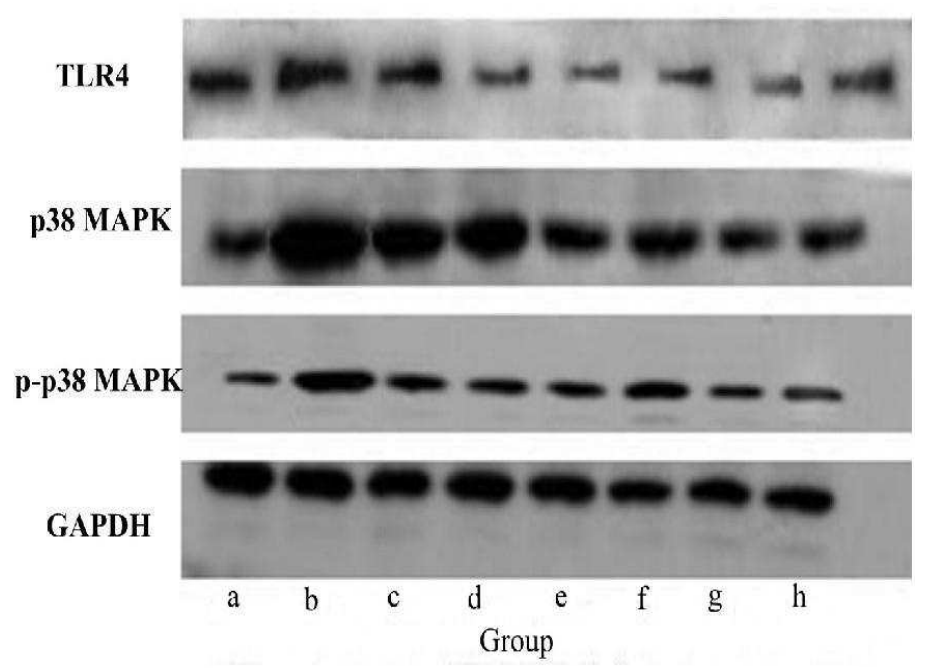

Figure 3: Western blot analysis of proteins involved in p38 MAPK signal pathway in hepatocytes

a: Negative control group (NC), b: Model control group (MC), c: High-dose CSS group (H-CSS), d: Low-dose CSS group (L-CSS), e: High-dose 
http://dx.doi.org/10.4314/ajtcam.v11i1.34

SLBZS group (H-SLBZS), f: Low-dose SLBZS group (L- SLBZS), g: High-dose integrated recipe group (H-IG), h: Low-dose integrated recipe group (L-IG).

\section{Discussion}

NASH is a popular chronic liver disease, and it has been one of the important factors resulting in hepatocirrhosis and liver cancer (Cohen et al., 2011; Fuchs et al., 2012). Though its etiology of NASH is still remaining unclear, it is considered to be associated with insulin resistance, lipid metabolic disorders, oxidative stress, lipid peroxidation or secretion of inflammatory cytokines. According to the two hit hypothesis, the first hit is fat accumulation in liver hepatocytes, which could lead to hepatic steatosis, further as a result of insulin resistance or lipid metabolic disorders. The second hit is exacerbating factors such as oxidative stress, lipid peroxidation or secretion of inflammatory cytokines which lead to hepatocyte inflammation, fibrosis and even hepatocirrhosis (Day et al., 1998).

Table 4: Expression of TLR4, p-p38 MAPK and p38 MAPK protein in hepatocytes

\begin{tabular}{|l|c|c|c|c|}
\hline Group & TLR4 / GAPDH & $\mathrm{p}$-p38 MAPK / GAPDH & $\mathrm{p} 38$ MAPK / GAPDH & $\begin{array}{c}\mathrm{p}-\mathrm{p} 38 \mathrm{MAPK} / \\
\mathrm{p} 38 \mathrm{MAPK}\end{array}$ \\
\hline NC & $0.85 \pm 0.11$ & $0.40 \pm 0.02$ & $1.55 \pm 0.20$ & $0.26 \pm 0.02$ \\
\hline MC & $1.40 \pm 0.10^{\mathrm{a}}$ & $1.69 \pm 0.08^{\mathrm{a}}$ & $4.14 \pm 0.10^{\mathrm{a}}$ & $0.41 \pm 0.01^{\mathrm{a}}$ \\
\hline H-CSS & $0.86 \pm 0.09^{\mathrm{b}}$ & $0.75 \pm 0.03^{\mathrm{b}}$ & $2.64 \pm 0.09^{\mathrm{b}}$ & $0.28 \pm 0.002^{\mathrm{b}}$ \\
\hline L-CSS & $0.75 \pm 0.08^{\mathrm{b}}$ & $0.69 \pm 0.06^{\mathrm{b}}$ & $2.79 \pm 0.07^{\mathrm{b}}$ & $0.25 \pm 0.02^{\mathrm{b}}$ \\
\hline H-SLBZS & $0.70 \pm 0.04^{\mathrm{b}}$ & $0.66 \pm 0.06^{\mathrm{b}}$ & $2.38 \pm 0.08^{\mathrm{b}}$ & $0.28 \pm 0.016^{\mathrm{b}}$ \\
\hline L-SLBZS & $0.76 \pm 0.03^{\mathrm{b}}$ & $0.91 \pm 0.04^{\mathrm{b}}$ & $2.52 \pm 0.13^{\mathrm{b}}$ & $0.36 \pm 0.003^{\mathrm{c}}$ \\
\hline H-IG & $0.64 \pm 0.03^{\mathrm{b}}$ & $0.46 \pm 0.03^{\mathrm{b}}$ & $2.03 \pm 0.40^{\mathrm{b}}$ & $0.23 \pm 0.031^{\mathrm{b}}$ \\
\hline L-IG & $1.09 \pm 0.13^{\mathrm{c}}$ & $0.67 \pm 0.02^{\mathrm{b}}$ & $2.09 \pm 0.09^{\mathrm{b}}$ & $0.32 \pm 0.004^{\mathrm{b}}$ \\
\hline
\end{tabular}

Values are means \pm S.D. of 6 rats per group; ${ }^{a} P<0.01$ compared with negative control group; ${ }^{\mathrm{b}} P<0.01,{ }^{\mathrm{c}} P<0.05$ compared with model control group

In the present research, HFD induced rat model of NASH successfully replicated several typical histopathological characteristics of NASH in human, such as hepatic lipid accumulation, hepatocyte steatosis and inflammatory cell infiltration. It was consistent with the previous study (Yang et al., 2011; Ji et al., 2011). We showed that CSS and SLBZS decreased the serum level of TG, and ameliorated the symptoms of inflammation in NASH model rats. Thus, further study is needed to elucidate the anti-inflammatory mechanism of CSS and SLBZS. We determined several proteins involved in p38 MAPK signal pathway which were closely related with inflammatory reaction and apoptosis (Wagner et al., 2009). The results demonstrated that activation of TLR4-p38 MAPK signal pathway in hepatocytes was involved in the development of NASH. CSS and SLBZS successfully suppressed the activation of TLR4, p-p38 MAPK and p38 MAPK involved in p38 MAPK signal pathway.

In conclusion, this study revealed that CSS and SLBZS might work as a significant anti-inflammatory effect on hepatocyte of NASH induced by HFD by inhibiting the activation of TLR4, p-p38 MAPK and p38 MAPK involved in p38 MAPK signal pathway. CSS and SLBZS may be a potentially alternative and complementary medicine to protect against liver injury, alleviate the inflammation reaction, moderate NASH progression. However, some limitation of the present study should be further to explored whether the high-dose Chinese medicines have any side effects or be in an acceptable margin of safety, and which elements of the Chinese medicines have these special therapeutical effects.

\section{Acknowledgements}

The present work was supported by a grant (No.30973694) from Natural Science Foundation of China.

\section{References}

1. Alisi, A., Panera, N., and Nobili, V. (2010). Toll-like receptor 4: A starting point for proinflammatory signals in fatty liver disease. Hepatology, 51: 714-715.

2. Angulo, P. (2002). Nonalcoholic fatty liver disease. N EngI J. Med. 346:1221-1231. 
http://dx.doi.org/10.4314/ajtcam.v11i1.34

3. Chen, L., Zhu, J. S., and Ren, J. P. (2004). Protective and curative effects of Chaihu Shugan San (CHSGS) on acute liver injury caused by carbon tetrachloride in rats. Chin J. Integr. Med. Liver Dis., 14: 42-43.

4. Chiang, L. C., Ng, L. T., Liu, L. T., Shieh, D. E., and Lin, C. C. (2003). Cytotoxicity and anti-hepatitis B virus activities of saikosaponins from Bupleurum species. Planta Med., 69: 705-709.

5. Choi, S., Diehl, and A. M. (2005). Role of inflammation in nonalcoholic steatohepatitis. Curr. Opin. Gastroenterol., 21: $702-707$.

6. Cohen, J. C., Horton, J. D., and Hobbs, H. H. (2011). Human fatty liver disease: Old questions and new insights. Science, 332: $1519-1523$.

7. Day, C. P., and James, O. F. (1998). Steatohepatitis: A tale of two "hits"?. Gastroenterology, 114: 842-845.

8. Fu, D. C., Yang, S. Z., Song, X. F., and Xiao, Y. (2007). Effect of Chaihu Shugan San in treating liver fibrosis. Chin J. Gerontol., 27: 1146-1148.1. Alisi, A., Panera, N., and Nobili, V. (2010). Toll-like receptor 4: A starting point for proinflammatory signals in fatty liver disease. Hepatology, 51: 714-715.

9. Fuchs, M. (2012). Non-alcoholic fatty liver disease: The bile acid-activated farnesoid x receptor as an emerging treatment target. J. Lipids, 2012: 934396.

10. Ge, H. Y., Chen, B., Liu, H. L., and Li, H. Q. (2011). Protective effect of Bupleurum Saikosaponin on alcoholic liver disease in rats. Chin J Gerontol., 31: $662-663.2$.

11. Hamelet J., Noll C., Ripoll C., Paul J. L., Janel N., Delabar J. M. (2009). Effect of hyperhomocysteinemia on the protein kinase DYRK1A in liver of mice. Biochem. Biophys. Res. Commun., 378: 673-677.

12. Ji, G. Y., Yang, Q. H., Hao, J., Guo, L. N., Chen, X., Hu, J. P., Leng, L., and Jiang, Z. Q. (2011). Anti-inflammatory effect of genistein on non-alcoholic steatohepatitis rats induced by high fat diet and its potential mechanisms. Int. Immunopharmacol., 11: 762-768.3.

13. Jin, M. S., and Lee, J. O. (2008). Structures of the toll-like receptor family and its ligand complexes. Immunity, 29: 182-191.

14. Kleiner, D. E., Brunt, E. M., Natta, M. V., Behling, C., Contos, M. J., Cummings, O. W., Ferrell, L. D., Liu,Y. C., Torbenson, M. S., Unalp-Arida, A., Yeh, M., McCullough, A. J., and Sanyal, A. J. (2005). Design and validation of a histological scoring system for nonalcoholic fatty liver disease. Hepatology, 6: $1312-1321$.

15. Kopec, K. L., and Burns, D. (2011). Nonalcoholic fatty liver disease: a review of the spectrum of disease, diagnosis, and therapy. Nutr. Clin. Pract., 26: $565-576$.

16. Kudo, H., Takahara, T., Yata, Y., Kawai, K., Zhang, W., and Sugiyama, T. (2009). Lipopolysaccharide triggered TNF- $\alpha$-induced hepatocyte apoptosis in a murine non-alcoholic steatohepatitis model. J. Hepatol., 51: 168-175.

17. Lalor, P. F., Faint, J., Aarbodem, Y., Hubscher, S. G., and Adams, D. H. (2007). The role of cytokines and chemokines in the development of steatohepatitis. Semin. Liver Dis., 27: 173-193.

18. Li, S. Q., Su, Z. H., Peng, J. B., Zou, Z. M., and Yu, C. Y. (2010). In Vitro and in Vivo Antioxidant Effects and the Possible Relationship between the Antidepression Efficacy of Traditional Chinese Medicine Formulation Chaihu Shugan San. Chin J. Nat. Med., 8: $353-361$.

19. Li, Y. G., Ji, D. F., Zhong, S., Zheng, X. L., and Shi, L. G. (2011). Protective effect of saponins extracted from Panax japonicus on ethanol-induced hepatic cells L-O2 injury. Chin J. Pharmacol. \& Toxicol., 25: 289-295.

20. Li, Z. H., Wang, J., Wang, Y. W., Cai, R. L., Sun, J., Ye, M. G., (2012). Effect of Shenlingbaizhu powder on serum levels of EGF, SOD and MDA in ulcerative colitis rats with syndrome of dampness stagnancy due to spleen deficiency. World Chin J. Dig., 20, 410-413.

21. Liu, X. P., Jia, Y. X., Liu, Q., and Wu, J. J. (2011). Effects of Shenglingbaizhusan decoction on CD44, D62p and CD54 in ulcerative colitis rats with asthenia of spleen. Lishizhen Medicine and Materia Medica Res., 22: 2671-2673.

22. Liu, Y., Zhang, Z., Wang, L., Li, J., Dong, L., Yue, W., Chen, J., Sun, X., Zhong, L., and Sun, D. (2010). TLR4 monoclonal antibody blockade suppresses dextran-sulfate-sodium-induced colitis in mice. J. Gastroenterol. Hepatol., 25: 209-214.

23. Liu, Y. L., and Lv, J. H. (2012). Hypolipidemic and antioxidative effect and hepatic protective effect of total glucosides of peony's on non-alcoholic fatty liver. J. Hainan Medical College 18: 158-161.

24. Luo, H. Y., Wang, Y. F., Zhai, S. S., Zhang, Y. J., Nan, X., Bai, C. X., Shi, S. S., Yu, C. Z., Yue, W., Kong, W., Pei, X. T. (2008). The function identification of hepatocyte differentiated from CTLA4-Gene modified bone marrow mesenchymal stem cells. Progress in biochemist and biophysics., 35: 1021-1030.

25. Pang, F. M., Zhang, D. X., and Huang, J. R. (2009). Effect of Chaihu Shugan San on B ultrasonic and the index of liver fibrosis in nonalcoholic fatty liver disease patient. Sichuan J. Tradit. Chin. Med., 27: 66-67.

26. Takeda K., and Akira S. (2004). TLR signaling pathways. Semin. Immunol., 16: 3-9.

27. Tian, J., Wang, Y., He, Z., Gao, Y., Rundhaug, J. E., and Wang, X. (2011). Hydroxyethyl starch (130 kD) inhibits toll-like receptor 4 signaling pathways in rat lungs challenged with lipopolysaccharide. Anesth. Analg., 113: 112-119.

28. Tilg, H., Diehl, and A. M. (2000). Cytokines in alcoholic and nonalcoholic steatohepatitis. N Engl J. Med., 343: $1467-1476$. 
http://dx.doi.org/10.4314/ajtcam.v11i1.34

29. Tilg, H. and Moschen, A. R. (2010). Evolution of inflammation in nonalcoholic fatty liver disease: The multiple parallel hits hypothesis. Hepatology, 52: 1836-1846.

30. Vanni, E., Bugianesi, E., Kotronen, A., De, M. S., Yki-Järvinen, H., and Svegliati-Baroni, G. (2010). From the metabolic syndrome to NAFLD or vice versa? Dig. Liver Dis., 42: 320-330.

31. Wagner, E. F., and Nebreda, A. R. (2009). Signal integration by JNK and p38 MAPK pathways in cancer development. Nat. Rev. Cancer, 9: 537-549.

32. Wang, C. H., Geng, Q. G., and Wang, Y. X. (2012). Protective effect of atractylenolide I on immunological liver injury. J. Chinese Materia Medica, 37: 1809-1812.

33. Xu, B., Zhang, Y. T., Xu, X. G., Wang, D. J., Xu, T. D., and Li, D. Y (2010). Role of Toll-like receptor 4/MAPKs pathway on monocyte chemoattractant protein-1 secretion induced by oxidized low density lipoprotein in vascular smooth muscle cells. Chin J. Pathophysiol., 26: 848-852.

34. Xue, C. R., Zhou, and X. L. (2010). Glycometabolism in chronic pancreatitis and intervening effect of Chaihu Shugan San. Chin J. Surg. Integr. Tradit. Western Med., 16: 189-191.

35. Yang, Q. H., Hu, S. P., Zhang, Y. P., Xie, W. N., Li, N., Ji, G. Y., Qiao, N. L., Lin, X. F., Chen, T. Y., and Liu, H. T. (2011). Effects of berberine on expressions of uncoupling protein-2 mRNA and protein in hepatic tissue of non-alcoholic fatty liver disease in rats. Chin J. Integr. Med., 17: $205-211$.

36. Yimin, Furumaki, H., Matsuoka, S., Sakurai, T., Kohanawa, M., Zhao, S., Kuge, Y., Tamaki, N., and Chiba, H. (2012). A novel murine model for non-alcoholic steatohepatitis developed by combination of a high-fat diet and oxidized low-density lipoprotein. Lab. Invest., 92: $265-281$.

37. You, Y., Liu, Y. H., and Gao, S. L. (2012). Effect and mechanism of Shenling Baizhu San on the murine model of inflammatory bowel disease induced by dextran sodium sulfate in mice. Chin J. Exp. Tradit. Med. Form., 18: 136-140.

38. Zeng, J., Li, F., Jia, X. M., and Li, C. Y. (2011). Comparison of the effects of Ginsenoside Rg3 and IFN-alpha on hepatic fibrosis induced by schistosomiasis japonica in mice. J Pathog. Biol., 6: 825-827.

39. Zhang, J., Liu, X. N., Zhang, P. J., Su, H., Lang, J., Zhuang, Z. N., Meng, Z. L., and Wu, X.Y. (2011). Effect of atractylodes macrocephalaon polysaccharide pre-disposal treatment after ischemia reperfusion injury of liver on the chondriosome structure of hepatic cell in rats. J. Hepatopancreatobiliary Surg., 23: 4-6.

40. Zhao, R. X., Zheng, L. Y., Pan, J. Q., Chen, Y. Y., Lv, J. H., Zhou, Y. B., Pan, W. S., and Xiao, Y. (2012). The antioxidative mechanism of total glucosides of paeony on protection against the fatty liver in NAFLD rats. J. Guangdong Pharmaceutical University 28: 430-434.

41. Zheng, C. W., Ding, H. M., Chen, Y., and Feng, Y. B. (2011). Experimental study of saikosaponin on hepatic fibrosis in rats. J Emerg. Tradit. Chin. Med., 20: 755.

42. Zhou, W., Hu, Y., Zhang, H. W., Shen, X. Y., Huang, Y. L., Tang, Z. J., and Hou, A. J. (2009). Effect of carboxymethylpachymaran on signal-transduction of TGF beta-Smad on hepatic fibrosis in rats. Chin J. EthoMed. Ethnopharm., 20: 16-18. 